\title{
THE GENERATION OF BUILDING FLOOR PLANS USING PORTABLE AND UNMANNED AERIAL VEHICLE MAPPING SYSTEMS
}

\author{
G. J. Tsai ${ }^{\text {a, }}$, Y. L. Chen ${ }^{\text {a }}$, K. W. Chiang ${ }^{\text {a }}$, Y. C. Lai ${ }^{\text {b }}$ \\ ${ }^{a}$ Dept. of Geomatics Engineering, National Cheng-Kung University, No. 1, Daxue Road, East District, Tainan, Taiwan - \\ tpp1114@gmail.com \\ b Dept. of Aerospace and Systems Engineering, Feng Chia University 100, Wenhwa Road, Seatwen, Taichung, Taiwan - \\ yingclai@fcu.edu.tw
}

Commission IV, WG IV/7

KEY WORDS: Location-Based Services (LBS), Floor Plan, Robot Mapping, Unmanned Aerial Vehicles (UAV), Portable Mapping System, Map Rectification, Geo-referenced

\begin{abstract}
:
Indoor navigation or positioning systems have been widely developed for Location-Based Services (LBS) applications and they come along with a keen demand of indoor floor plans for displaying results even improving the positioning performance. Generally, the floor plans produced by robot mapping focus on perceiving the environment to avoid obstacles and using the feature landmarks to update the robot position in the relative coordinate frame. These maps are not accurate enough to incorporate to the indoor positioning system. This study aims at developing Indoor Mobile Mapping System (Indoor MMS) and concentrates on generating the highly accurate floor plans based on the robot mapping technique using the portable, robot and Unmanned Aerial Vehicles (UAV) platform. The proposed portable mapping system prototype can be used in the chest package and the handheld approach. In order to evaluate and correct the generated floor plans from robot mapping techniques, this study builds the testing and calibration field using the outdoor control survey method implemented in the indoor environments. Based on control points and check points from control survey, this study presents the map rectification method that uses the affine transformation to solve the scale and deformation problems and also transfer the local coordinate system into world standard coordinate system. The preliminary results illustrate that the final version of the building floor plan reach 1 meter absolute positioning accuracy using the proposed mapping systems that combines with the novel map rectification approach proposed. These maps are well geo-referenced with world coordinate system thus it can be applied for future seamless navigation applications including indoor and outdoor scenarios.
\end{abstract}

\section{INTRODUCTION}

In the past few years, Mobile Mapping System (MMS) has been widely used in Geomatics engineering. Using various mapping sensors, such as Light Detection And Ranging (LiDAR), camera and high resolution visible sensors, geospatial information can be easily acquired by a mobile platform. The most common mobile mapping systems work by capturing more than one image which include the same feature point, captured from different perspective, allowing the $3 \mathrm{D}$ spatial information of objects to be calculated and measured with respect to the mapping frame (Tao, 2001). MMS is also being used with various vehicles, such as automobiles, aircraft, water-based vessels and Unmanned Aerial Vehicles (UAV). UAV was introduced in the beginning of the 19th century for applications of military mission and now are the popular platform for commercial applications or mass-market products. For the mapping techniques, an UAV enables to be used in some areas which are inaccessible to the traditional robot or land vehicle and also has a great potential for indoor mapping and navigation due to its flexibility and feasibility. The UAV borne MMS applications concentrate on monitoring, security tasks, surveillance, and remote sensing (Eisenbeiss, 2004); UAVPhotogrammetry mapping systems not only provide the 3D geospatial data, but also contribute to $3 \mathrm{D}$ model generation (Neitzel, 2012). Most UAV mapping systems incorporate the Global Positioning System/Inertial Navigation System (GPS/INS) and photogrammetric systems. With the rapid development of $\mathrm{UAV}$, the mapping system is poised to move into high accuracy
3D modular measurement systems. Low-cost laser scanner and Micro-Electro-Mechanical Systems (MEMS) GPS/ INS are the core roles of unmanned aerial system (UAS) (Jaakkola, 2010). More recently, the use of MMS has not been restricted to being mounted on mobile machines. A portable MMS has been proposed which is composed of a Position and Orientation System (POS) module working in conjunction with an image acquisition module, trigger control and data logging module (Chu, 2013). This system could be used in some areas that are inaccessible to vehicles.

Because of the increasing demand for accurate indoor maps, MMS has evolved to the next phase: indoor environment. This application would be useful for emergency, indoor navigation, as well as Location-Based Services (LBS), etc. In fact, high accuracy map of indoor environments, once it caught on, would soon become indispensable in indoor navigation and LBS. In general, building blueprints are not easy to access; determining and evaluating the absolute accuracy of these blueprints are a logistical nightmare. In robotics research, most robotic navigation tasks are based on the building maps, therefore, methods for generating relatively reliable floor plans has become an important topic (Okorn, 2010). Robot mapping systems focus on real time localization, sensing and measuring the environment at the same time; as a result, Simultaneously Localization and Mapping (SLAM) algorithms are widely used in robot applications.

\footnotetext{
* Corresponding author
} 
After analyzing previous researches, most robot mapping systems concentrate on developing high efficient SLAM algorithms or using different platforms to implement the SLAM. However, these floor plans generated by the robot mapping are limited to certain mapping system and therefore, lack of generalization. Even though the maps are produced by the same system, it requires extra works to relocate the position and reorientation manually in order to reuse the produced maps. Besides, floor plans generated by the SLAM are hard to evaluate the accuracy and can't directly be used in other applications. The main problem is that these floor plans are not in the standard coordinate frame and therefore it is tough to use. This study proposes the modules for generating indoor floor plan that can transfer the local map reference in robot system into well georeferenced map with global coordinate system. This kind of map can be applied for various seamless applications, such as the navigation system from outdoor to indoor. There are four kinds of platforms, robot, handheld approach, chest package and UAV applied in this study. The experiment adopted the handheld approach and chest package we designed to compare with UAV and ground robot. To evaluate the absolute positioning accuracy and rectify the indoor floor plans from different platforms, this study built the testing and calibration field based on the control survey to analyze each result for purposes of cross validation.

\section{METHODOLOGY}

SLAM has been developed for many years, and most algorithms achieve the robust performance on mapping system as well as localization. The generated map often used in navigating the robot and implementing the autonomous motion planning. In this study, the issued problem is that if user wants to update the map or implement the motion planning with known map, it needs to take extra efforts to initialize the position and orientation. It is also known as global localization problem that puts the robot into the known environment and has to localize itself from scratch. The primary issue is that the map is often generated in different reference frames. This problem also occurs when users apply the robot map on different applications. Another problem is that without any landmarks or absolutely aiding information, the accuracy of robot mapping is degraded with time. Therefore, this study proposes the novel map rectification process to rectify and transfer the raw map into the global frame with reliable scale and accuracy. Figure 1 shows the floor plan generalization processes and the detail is described below.

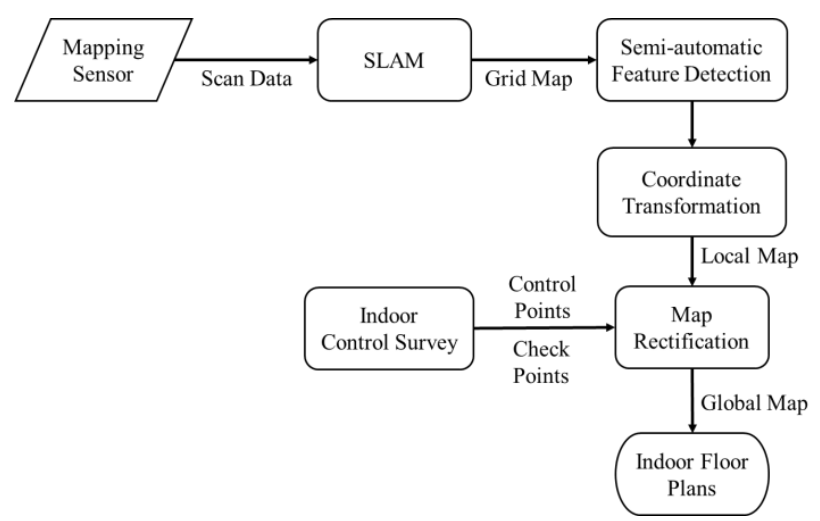

Figure 1. The flow chart of floor plans generalization

\subsection{Hector SLAM}

SLAM is a common solution that senses and recreates the map of an unknown environment and simultaneously tracks the position and attitude of the platform in robot research. The core integrating algorithms are classified into three paradigms, Kalman filters, particle filters and graph-based processing. In SLAM applications, most of algorithms rely on environmental landmarks and the sensors' raw-data which are integrated into the SLAM model. For the robot map, occupancy grid maps cut the environment into the small cells, each of which includes different attributes to represent the environment. An occupancy grid maps record the whole environment information that sensors observe and transfer it into the occupancy grid. This study aims at using grid-based SLAM algorithms, Hector SLAM, to generate the floor plans.

Due to the rapid development of mapping and localization sensors, most robots are equipped with a payload of a small laser scanner and MEMS IMU. Hector SLAM is one of the SLAM algorithms developed for laser scanners (Kohlbrecher, 2011). The main differences between these two SLAM algorithms are the 6 DOF motion estimation without odometry and scanmatching method. Figure 2 shows a flow chart of Hector SLAM process. This means the mapping system doesn't have to be mounted on at mobile robot; it could be mounted on UAV, human or other platforms without odometer.

The estimated pose information is an important parameter for scan-matching; it improves the performance. In addition, scanmatching also gives the posterior information to enhance the navigation results. Scan-matching plays a significant role in Hector SLAM. In the process, the data from the laser scanner is aligned with the map or other observations. Based on the high accuracy of laser scanners, with low distance measurement noise and high scan rates, scan-matching provides $2 \mathrm{D}$ pose estimation for the navigation filter by registering each piece of scanned data and aligning it with the existing map. This approach is based on the optimization of aligning the endpoint of the scan data and the existing map. The basic idea is follows the Gauss-Newton approach from (Lucas, 1981).

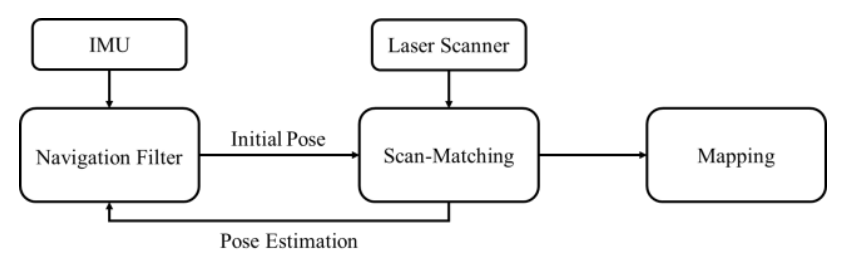

Figure 2. The data process of Hector SLAM

\subsection{Semi-automatic Feature Detection}

In this study, we adopt the gird-based SLAM algorithm which uses the occupancy grid map to represent the map. The occupancy grid map is the raster data that records the spatial information in each cell. However, the vector data is more suitable to present the floor plan due to the advantages of plotting and resolution. The grid map is limited by size of cell and the raster data cannot clearly displays the boundary of building. To transfer the raster data to the vector data, the edge and line feature detection is used in this study based on the covariance model (Van der Heijden, 1995). Compared with the sketch map, the redundant features like barriers or wrong feature lines are removed and the floor plan is generated only with the major margin. 


\subsection{Coordinate Transformation}

As described in section 2.2, the generated maps are located at different coordinate systems. To evaluate and process the different maps, it is necessary to transfer different maps into the same coordinate system using the local to local transformation. Besides, extracted maps and traditional control surveys are based on the planimetric surveying which has the meter unit of position. The global coordinate system uses the latitude and longitude in degree to present the position. In order to achieve the seamless application, this study uses the indoor control survey to build the relationship between local and global coordinate system.

Figure 3 presents the local and global coordinate system. $p_{x, y, z}$ is a point in local frame using the meter as the unit of position and the original point of local frame is $\mathrm{P}$ with known coordinate in global frame. The primary transformations used in this study are described as follows.

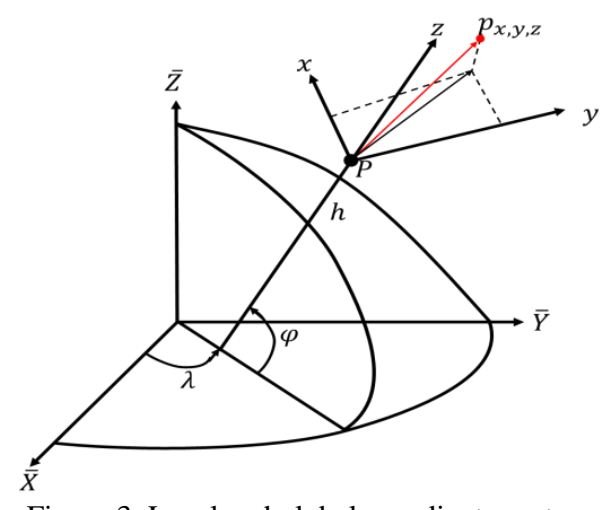

Figure 3. Local and global coordinate system

2.3.1 Local to Local Transformation: The generated maps from different algorithms or different systems always use different coordinate frames to present the results. In this study, local to local transformation is the $2 \mathrm{D}$ conformal transformation that used translation, rotation and scale parameters to transfer the maps in the same coordinate frame. This transformation is also used in the evaluation process and map rectification.

$$
\begin{aligned}
& x^{\prime}=s \times(\cos \alpha \cdot x+\sin \alpha \cdot y)+T_{x} \\
& y^{\prime}=s \times(-\sin \alpha \cdot x+\cos \alpha \cdot y)+T_{y}
\end{aligned}
$$

where $(x, y)$ is the raw coordinate, $\left(x^{\prime}, y^{\prime}\right)$ is the transferred coordinate, $s$ is the scale parameter that is equal to 1 due to the same unit of $\mathrm{x}$ and $\mathrm{y}$ axes,$\alpha$ is the rotation angle, $T_{x}$ and $T_{y}$ are translation parameters

2.3.2 Global to Local Transformation: The global to local transformation is applied in order to convert the control points measured from control surveys into local frame. These points with two coordinate systems can be used to build the conversion relationship between local and global. As Figure 3 shows, global coordinate can be represented in the form as longitude, latitude and ellipsoid height, $(\lambda, \varphi, \mathrm{h})$; and Cartesian coordinate frame of Earth-Centered, Earth-Fixed (ECEF), $(\bar{X}, \bar{Y}, \bar{Z})$. The first transformation process is transferring $(\lambda, \varphi, \mathrm{h})$ to $(\bar{X}, \bar{Y}, \bar{Z})$. The equation is formed as :

$$
\left[\begin{array}{l}
\bar{X} \\
\bar{Y} \\
\bar{Z}
\end{array}\right]=\left[\begin{array}{c}
(N+h) \times \cos \varphi \cos \lambda \\
(N+h) \times \cos \varphi \sin \lambda \\
\left(N\left(1-e^{2}\right)+h\right) \cos \varphi
\end{array}\right]
$$

where $\lambda$ is longitude, $\varphi$ is latitude, $N$ is the prime vertical and $e$ is the first numerical eccentricity referenced World Geodetic System 84 (WGS84).

After acquiring the $(\bar{X}, \bar{Y}, \bar{Z})$ from Equation (3), the local coordinate can be calculated by $R$ which includes two rotations to align ECEF and local frames. The transformation equation is formed as :

$$
\begin{gathered}
{\left[\begin{array}{l}
x \\
y \\
Z
\end{array}\right]=R \times\left[\begin{array}{l}
\bar{X} \\
\bar{Y} \\
\bar{Z}
\end{array}\right]} \\
R=\left[\begin{array}{ccc}
-\sin \lambda & \cos \lambda & 0 \\
-\cos \lambda \sin \varphi & -\sin \lambda \sin \varphi & \cos \varphi \\
\cos \lambda \cos \varphi & \sin \lambda \cos \varphi & \sin \varphi
\end{array}\right]
\end{gathered}
$$

2.3.3 As Figure 6 shows, global coordinate can be represented in the form as longitude, latitude and ellipsoid height, $(\lambda, \varphi, \mathrm{h})$; and Cartesian coordinate frame of Earth-Centered, Earth-Fixed (ECEF), $(\bar{X}, \bar{Y}, \bar{Z})$.The first transformation process is transferring $(\lambda, \varphi, \mathrm{h})$ to $(\bar{X}, \bar{Y}, \bar{Z})$. The equation is formed as:

2.3.4 Local to Global Transformation: After the local map is generated and rectified, the local map is transferred into the global coordinate system to be presented on the Google Earth. From local frame to global frame, the first step is to transfer the local $(\mathrm{x}, \mathrm{y}, \mathrm{z})$ to global $(\bar{X}, \bar{Y}, \bar{Z})$ that inverses the Equation (4) given by:

$$
\left[\begin{array}{l}
\bar{X} \\
\bar{Y} \\
\bar{Z}
\end{array}\right]=R^{\prime} \times\left[\begin{array}{l}
x \\
y \\
Z
\end{array}\right]
$$

To transfer $(\bar{X}, \bar{Y}, \bar{Z})$ to $(\lambda, \varphi, \mathrm{h})$, the usual procedure is to iterate beginning with the assumption to calculate the initial value. The specific iteration method can be referenced from (Kaplan, 1996).

\subsection{Indoor Control Survey}

Control surveys are used to establish the high precision of horizontal and vertical positions, and are also essential to reference framework for giving locations of data as well as the basis of originating or checking for subordinate surveys (Wolf, 2006). The key concept of control surveys is to connect a series of consecutive points with the distance and orientation determined from observations. Based on the control points established from control survey, all terrain is able to be mapped and accomplished the positions of planimetric features. In this study, indoor control survey is proposed to build the high accuracy testing filed for evaluating absolute positioning accuracy and calibration field for map rectification. However, most control surveys are always built in local frame. In order to estimate the conversion relationship between local frame and global frame, initial points of indoor control survey are measured with the global coordinate using the Geodesy approaches. The detail processes of indoor control survey are descried as follows. 
2.4.1 Control Points Selection: First and foremost, it is important that arranging all of the control points to be widely distributed in testing field. The challenge for indoor control survey is that each control points must be visible between two consecutive points. In outdoor environment, it is easy to select the points that can be clearly observed between each other. However, there are a lot of barriers that influence the point's selection in indoor environment such as wall, stair and pillars. This study established a control survey field from outdoor into indoor, the outdoor control points are initial points for coordinate transformation to derive globe frame from WGS84. On the other hand, indoor control points play a significant role to measure the building features for evaluation and map rectification.

2.4.2 Outdoor surveying to indoor surveying: After selecting the control points, this study used the electronical GPS (eGPS) device to observe the initial points with WGS84 coordinate in outdoor environment. An important advantage of eGPS is that it provides the centimeter level accuracy for horizontal and vertical position in a short period. It is a real time positioning system that based on a Real Time Kinematic (RTK) technique to enhance the positioning accuracy from GPS, a major difference between eGPS and RTK is that the former uses the network Virtual Reference Station (VRS) instead of real base station. In this study, we use outdoor points as the initial points with the given azimuth and original position, then we measured the distance and angle information between each two consecutive points from outdoor to indoor. According to the control survey theory, each control point can be determined in local frame and transfer into global frame. After establishing the control points, the building's feature points can be determined as well. The detail mathematical equation is described in (Wolf, 2006).

\subsection{Map Rectification}

In order to deal with the scale and deformation problems of generated map from different mapping systems, this study proposes the new method to refine the map and also implements the coordinate transformation to generate the map in global frame. The flow chart is shown in the Figure 4. In the beginning, the local map and control points are located in the same coordinate system. For rectifying the map, map rectification uses the affine transformation that includes 6 parameters, two translations, two scale, rotation and shear. In this transformation, it needs to select at least three tie points from control survey in order to calculate 6 parameters and eliminate the deformation problem. Therefore, there is a significant correlation between tie points selection and the accuracy of rectified map. Tie points should be widely and uniformly placed at distorted area to control deformation. Finally, the rectified map $\left(x_{m}^{\prime}, y_{m}^{\prime}, z_{m}^{\prime}\right)$ is transferred into global coordinate $\left(\varphi_{m}, \lambda_{m}, h_{m}\right)$ by using the local to global transformation.

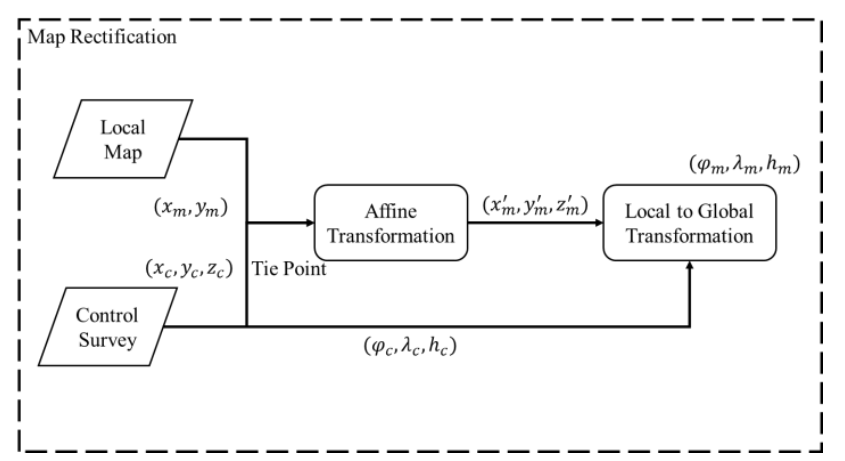

Figure 4 . The flow chart of map rectification

\section{EXPERIMENT}

Because the accuracy of the indoor map is not easy to assess and evaluate, this study adopts an indoor control survey for the mapping experiments. Before conducting the experiments, a control survey was established by using angle and distance measurements observed by a high accuracy device. A sketch of the control survey is shown in Figure 5; the pink triangular points represent the control points, and the red stars are the check points located in the building's corners for evaluating the floor plan. These check points measured by the control survey are represented as the building's boundary features to compare with floor plan. Total experimental area is about 1300 square meters, the width is about 30 meters and the length is about 60 meters.

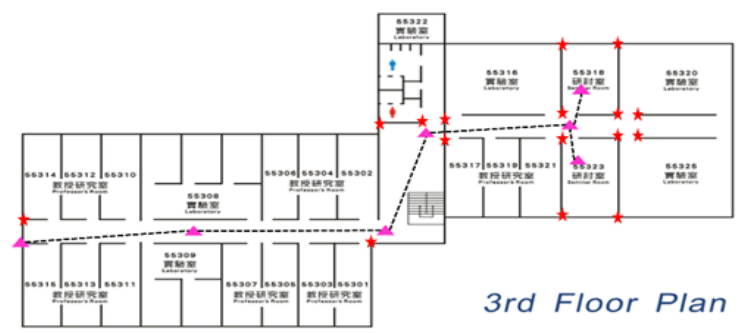

Figure 5. The sketch of indoor control points and check points

In this experiment, we deployed laser scanner (UST-20LX) on the different platforms, robot, UAV, handheld approach and chest package, to analyze and evaluate the performance of indoor floor plans. The configuration of commercial robot (Turtlebot) and UAV (AscTec Pelican) are shown in Figure 6.

Most of time, indoor mapping uses the ground robot to collect the data. In order to achieve the indoor MMS with more flexibility, this study proposes the two portable payloads for handheld approach and chest package to compare with other platforms. Although the robot is more stable, portable platform can avoid scanning the barriers such as chairs and tables. Handheld approach is a convenient way to require the indoor geospatial information that user holds the whole portable payload by hand and can accomplish whole experiments by oneself. Similar to handheld approach, the chest package uses the same device to generate the indoor floor plan. It is different from the platform that the device are allocated at square box and uses the shoulder strap to fix on the chest. Figure 7 shows the payload and actual operation for handheld approach and chest package.

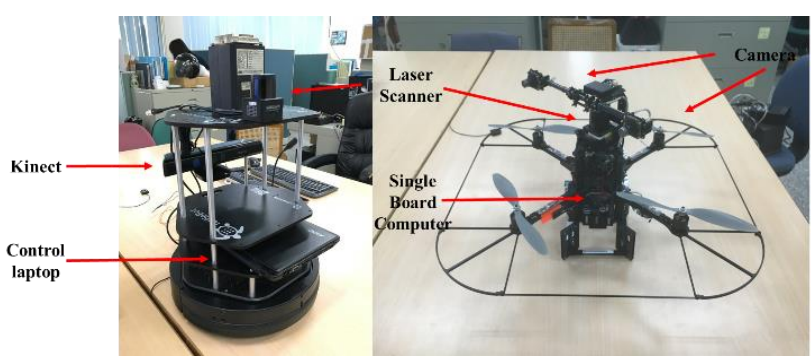

Figure 6. The configuration of mapping sensors on robot and UAV

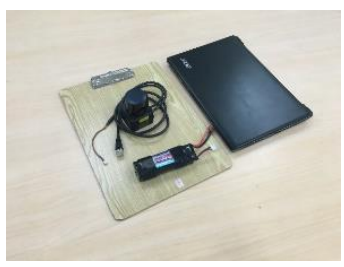

(a)

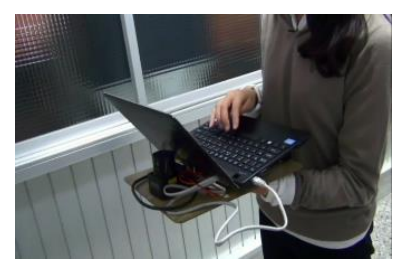

(b) 


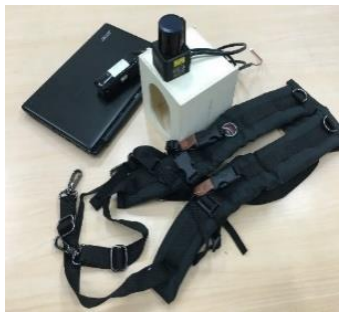

(c)

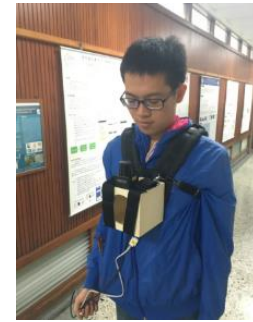

(d)
Figure 7. The indoor MMS using handheld approach and chest package. (a) shows payload for handheld approach; (b) shows actual operation for handheld approach; (c) shows payload for chest package and (d) shows actual operation for chest package

\section{RESULTS AND DISCUSSIONS}

Based on the previous experiments, the results of the indoor MMS are divided into two sections: the first section focuses on comparing the map results with four indoor MMS platforms, robot, UAV, handheld approach and chest package; the second section displays the indoor floor plans on Google Earth in global frame.

This section presents the floor plans generated by different platforms, robot, UAV, hand, and chest. In order to analyze the performance of each platform, it is necessary to transform the floor plan into the same coordinate system. Due to the mapping experiments were not conducted at the same time, each generated floor plan is not located in the same local coordinate. For analysis, the first process is to implement the local to local transformation using control points to transfer the maps into the same local frame. Figure 8 shows each generated maps using robot, UAV, handheld approach and chest package, respectively. The control points established from the indoor control survey are pink and the check points are red. The blue line shows the map results from different platforms and the green maps are the rectified results. It is obvious that raw maps which are presented in blue include the deformation problem after comparing with check points. The rectified results of each platforms is with better performance after map rectification.

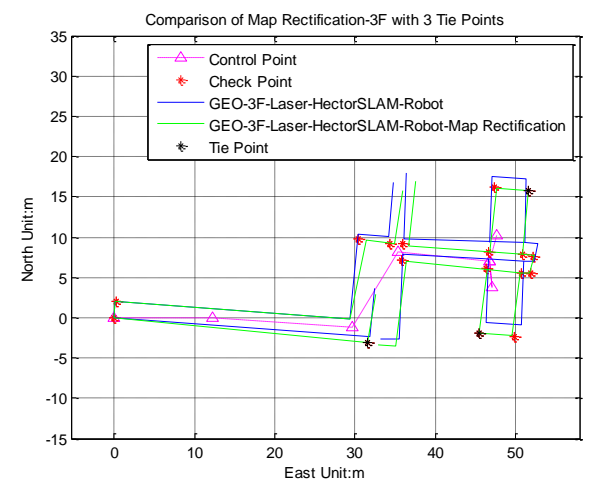

(a)

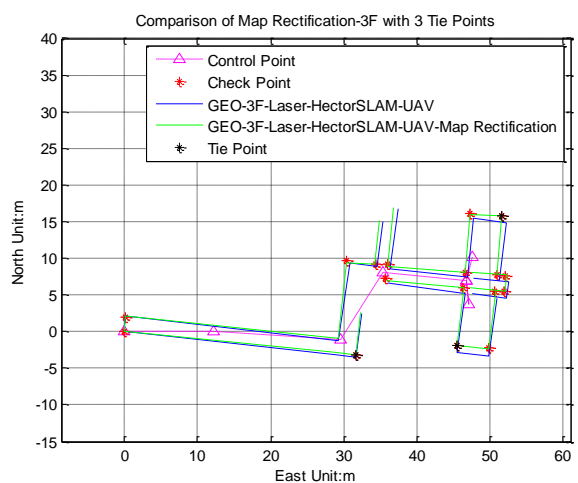

(b)

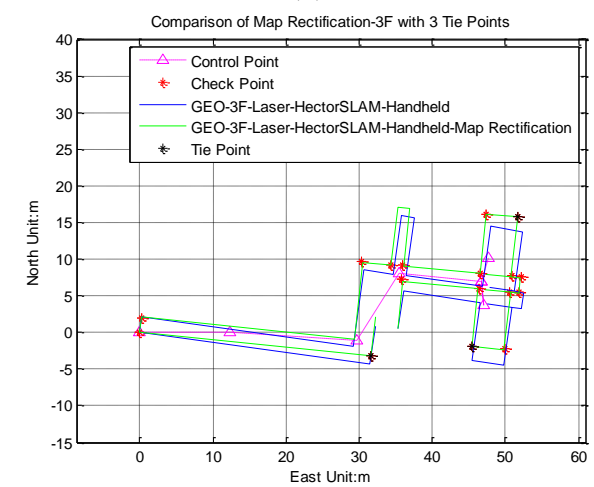

(c)

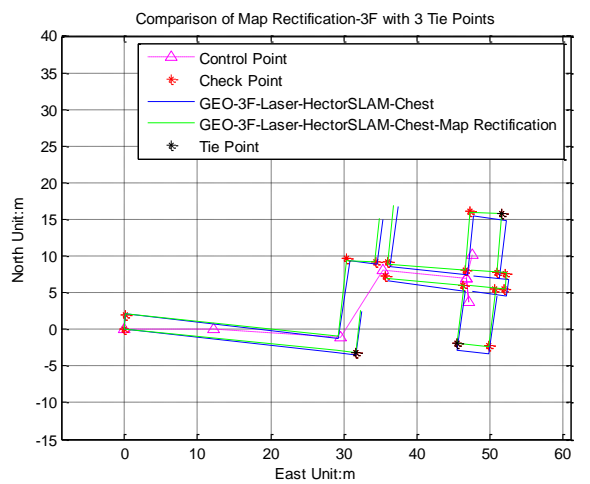

(d)

Figure 8. The generated map using different platforms after map rectification. (a) shows the map using robot; (b) shows the map using UAV; (c) shows the map using handheld approach and (c) shows the map using chest package

Figure 9 shows the error analysis in the bar chart, it is important that all error can be effectively controlled under $1 \mathrm{~m}$ using the proposed map rectification. From Table 1, the RMSE of each raw map is about 0.8 to 1.8 meters. The raw map using robot platform is better than other platforms. With map rectification, all RMSE and mean errors are under a meter and with the great improvement. The improvement of handheld approach, chest package, UAV and robot are 91\%, 91\%, $81 \%$ and $64 \%$, respectively. 


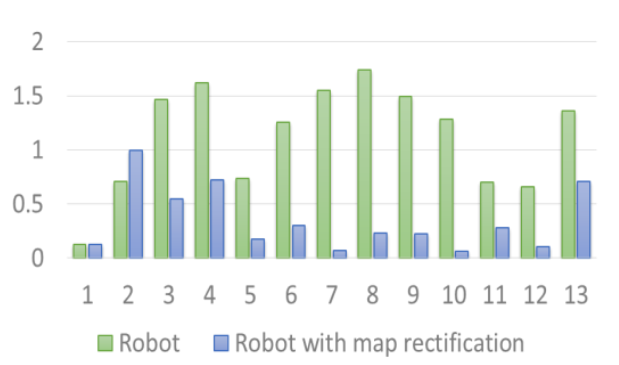

(a)

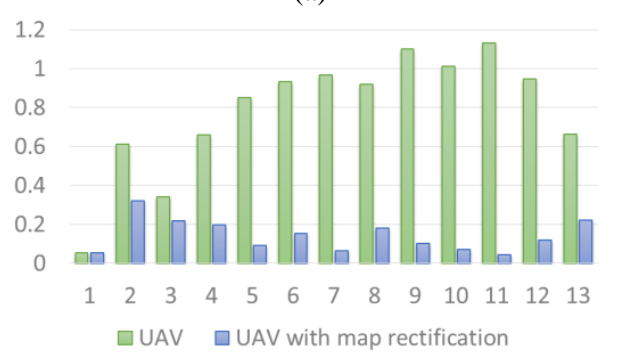

(b)

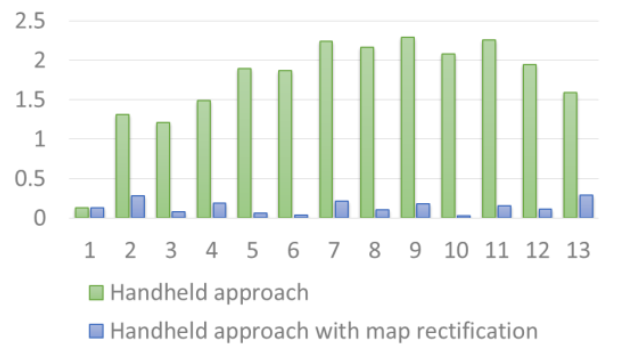

(c)

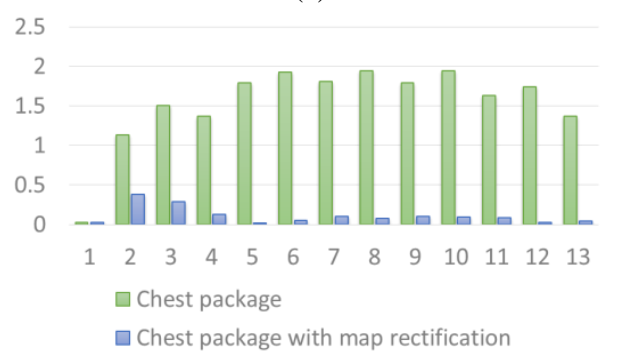

(d)

Figure 9. The error of feature points using different platforms with map rectification at $3 \mathrm{~F}$. (a) shows the error using robot; (b) shows the error using UAV; (c) shows the error using handheld approach and (d) shows the error using chest package

\begin{tabular}{|c|c|c|c|}
\hline Platform & $\begin{array}{c}\text { Mean Error } \\
\text { (Unit: } \mathrm{m})\end{array}$ & $\begin{array}{c}\text { RMSE } \\
(\text { Unit: } \mathrm{m})\end{array}$ & $\begin{array}{c}\text { Improvement } \\
(\%)\end{array}$ \\
\hline Robot & 1.145 & 1.257 & $64 \%$ \\
\hline $\begin{array}{c}\text { Robot } \\
\text { with map } \\
\text { rectification }\end{array}$ & 0.350 & 0.451 & $81 \%$ \\
\hline UAV & 0.783 & 0.838 & \\
\hline $\begin{array}{c}\text { UAV } \\
\text { with map } \\
\text { rectification }\end{array}$ & 0.141 & 0.162 & $91 \%$ \\
\hline $\begin{array}{c}\text { Handheld } \\
\text { approach }\end{array}$ & 1.730 & 1.813 & \\
\hline $\begin{array}{c}\text { Handheld } \\
\text { approach } \\
\text { with map } \\
\text { rectification }\end{array}$ & 0.144 & 0.165 & $91 \%$ \\
\hline
\end{tabular}

\begin{tabular}{|c|c|c|c|}
\hline Chest package & 1.548 & 1.621 & \\
\hline $\begin{array}{c}\text { Chest package } \\
\text { with map } \\
\text { rectification }\end{array}$ & 0.111 & 0.151 & $91 \%$ \\
\hline
\end{tabular}

Table 1. Evaluation of map rectification using different platforms

In robot mapping system, most indoor maps are located and presented in local coordinate system. This study proposed the map rectification to integrate the indoor control survey and coordinate transformation that transfers the local coordinate to the global coordinate system. Figure 10 shows the previous rectified maps on Google Earth in the format of longitude and latitude. As shown in Figure 10, rectified floor plans using Kinect are displayed in blue on Google Earth. The Figure 10 shows rectified floor plans using robot, UAV, handheld approach and chest package on Google Earth. As can be seen from this figures, the floor plans are placed in the building area. These indoor floor plans with the global coordinate can be used to connect the outdoor roadmap that is also located in the global coordinate system to achieve the seamless navigation.

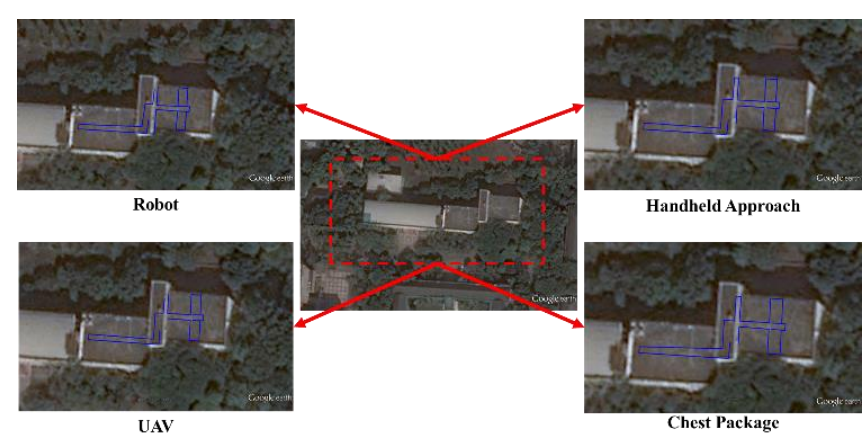

Figure 10. The generated map using different platforms after map rectification on Google Earth

\section{CONCLUSIONS}

In robot mapping, the generated map includes the scale and deformation problem. To achieve the a meter level accuracy of floor plans, this study constructs the novel data processing for generating the high accuracy floor map. Moreover, generated maps are transferred to the standard global coordinate system that can be used for seamless applications and different mapping systems.

In this study, we implemented four mapping systems, robot, UAV, handheld approach and chest package. The handheld approach and chest package are portable and are placed in human body. The results show that the generated floor plan using UAV is better than other platforms due to the higher stability. Finally, all of floor plans can achieve the one meter level accuracy and remove deformation problem with map rectification. The results clearly demonstrate that the proposed map rectification is effective for improving the map accuracy.

In addition to the improved map accuracy, the rectified floor plans displayed on Google Earth shown in the result section that these floor plans are good enough to be located in the experimental building within a reasonable range. It is worth mentioning that most of the floor plans generated from other system may not achieve this accuracy because of the scale and deformation problem. In order to accomplish the seamless and common applications, it is important to build the floor plan in the standard coordinate system. 


\section{ACKNOWLEDGEMENTS}

The author would acknowledge the financial supports provided by the Ministry of Science and Technology (MOST-104-2923M-006-001-MY3), and Aim for the Top University Project.

\section{REFERENCES}

Tao, V.C.; Li, J., 2001. Advances in mobile mapping technology. International Society for Photogrammetry and Remote Sensing (ISPRS) Book Series: Taylor and Francis Group: London, UK.

Eisenbeiss, H., 2004. A mini unmanned aerial vehicle (uav): System overview and image acquisition. In: Proceedings of the International Workshop on Processing and Visualization Using HighResolution Imagery, Pitsanulok, Thailand.

Neitzel, F.; Klonowski, J., 2012. Mobile 3d mapping with a lowcost uav system. In: International Conference on Unmanned Aerial Vehicle in Geomatics (UAV-g), Vol. XXXVIII-1/C22, pp $39-44$.

Chu, C.H.; Chiang, K.W.; Lin, C.A., 2013. The performance analysis of a portable mobile mapping system with different gnss processing strategies, Proceedings of the 26th International Technical Meeting of The Satellite Division of the Institute of Navigation (ION GNSS+ 2013), Nashville, TN, September, pp $689-703$

Okorn, B.; Xiong, X.; Akinci, B.; Huber, D., 2010. Toward automated modeling of floor plans, Proceedings of the Symposium on 3D Data Processing, Visualization and Transmission Paris, France, 5/17.

Grzonka, S.; Grisetti, G.; Burgard, W., 2009. Towards a navigation system for autonomous indoor flying. In Robotics and Automation. ICRA '09. IEEE International Conference on, IEEE: Kobe; pp 2878 - 2883.

Fallon, M. F.; Johannsson, H.; Brookshire, J.; Teller, S.; Leonard, J. J., 2012. Sensor Fusion for Flexible Human-Portable BuildingScale Mapping. Intelligent Robots and Systems (IROS), IEEE/RSJ International Conference on, IEEE: Vilamoura, pp 4405-4412.

Kohlbrecher, S.; von Stryk, O.; Meyer, J.; Klingauf, U., 2011. A flexible and scalable slam system with full $3 \mathrm{~d}$ motion estimation. In Safety, Security, and Rescue Robotics (SSRR), 2011 IEEE International Symposium on, IEEE: Kyoto; pp 155 - 160.

Grisetti, G.; Stachniss, C.; Burgard, W., 2007. Improved techniques for grid mapping with rao-blackwellized particle filters. Robotics, IEEE Transactions on 2007, 23, 34 - 46.

Kaplan, E.D., 1996. Understanding gps: Principles and applications. Artech House.

Wolf, P.R.; Ghilani, C.D., 2006. Elementary surveying: An introduction to geomatics. Pearson/Prentice Hall.

Van der Heijden, F., 1995. Edge and line feature extraction based on covariance models. Pattern Analysis and Machine Intelligence, IEEE Transactions on 1995, 17, 16-33. 